\title{
Characterization of Viability of Seeds by Using Dynamic Speckles and Difference Histograms
}

\author{
Margarita Fernández ${ }^{1}$, Adriana Mavilio ${ }^{1}$, Héctor Rabal ${ }^{2}$, and Marcelo Trivi ${ }^{2}$ \\ ${ }^{1}$ Instituto Superior Politécnico “José A. Echeverría”, Dpto. de Física, Facultad de \\ Ingeniería Eléctrica, Calle 114 s/n, Apartado 6028, Marianao 15, La Habana, Cuba. \\ mflimia@electrica.cujae.edu.cu, adriana@electrica.cujae.edu.cu \\ ${ }^{2}$ Centro de Investigaciones Ópticas (CIC-CONICET) and UID Optimo, Dpto. \\ Fisicomatemáticas, Facultad de Ingeniería, Universidad Nacional de La Plata, Casilla de \\ Correo 124, 1900 La Plata, Argentina \\ hrabal@volta.ing.unlp.edu.ar, marcelot@,ciop.unlp.edu.ar
}

\begin{abstract}
We propose the use of Difference Histogram method for classification of soya seed speckle images. The time history speckle patterns (THSP), obtained from the dynamic speckle patterns of the seeds were processed for texture classification based on seed vigor and viability. In this work, bean seeds were analyzed with different humidity levels, ie. at 15, 25, 35 and 45 minutes since the sample was taken out from the humid germination paper and submitted to the imaging process, with the aim of determining the influence of this temporal parameter in the classification result (dead or alive). The whole set of seeds was previously analyzed and classified in viable (alive) or not viable (dead) by experts in the matter by applying a traditional method. According to the obtained results the proposed method revealed to be appropriate for the task of classification of seeds. In the case of the highest humidity level the disagreement between our method and the conventional one was the greatest. It should be said that in this case the analyzed images were noisier.
\end{abstract}

\section{Introduction}

Dynamic speckle is a related phenomenon occurring when laser light is scattered by objects showing some type of activity [1]. This is the case of many biologic samples as seeds [2], fruits, etc., and some non biologic ones as corrosion phenomena and drying of paints [3].

In the activity images [3], corresponding to dynamic speckles, the size, shapes and spatial distribution of the areas of same gray tones, change with time. Characterization of seed quality can be done by taking into account vigor and viability of seeds through textural analysis of the time history of speckle patterns (THSP), obtained from the dynamic speckles [4]. In the THSP images, the rows represent different points on the object and the columns their intensity state in every sampled instant. The activity of the sample appears as intensity changes in the horizontal direction. So, when a phenomenon shows low activity (dead seeds), time variations of 
the speckle pattern are slow and the THSP shows elongated shapes. When the phenomenon is very active (alive seeds), the THSP resembles an ordinary granulated speckle diagram. Consequently, textural features provide information that allows the quantitative characterization of the seed state.

Many methods for texture analysis have been reported [5-8], such as statistical and spectral ones using digital filtering [9-11]. We propose a methodology based on the Histogram Difference Method developed by Unser in 1986 [7] to characterize texture of the THSP images. The images were analyzed through the Unser discrimination function which was computed by using the elements of the difference histogram [7] as texture features. This is the histogram of the image obtained by subtracting a shifted image from the original image.

In this work, bean seeds were analyzed with different humidity levels, ie. at 15, 25, 35 and 45 minutes since the sample was taken out from the humid germination paper and submitted to the imaging process. It was done in order to study the influence of this temporal parameter in the classification process (dead or alive). The whole set of seeds was previously analyzed and classified in viable or not by experts in the matter by applying a traditional method.

A similar procedure could be used to classify other kinds of images, corresponding to a dynamic speckle processes.

\section{Time-History Images of Dynamic Speckle Patterns (HTSP)}

In the optical experiment, dynamic speckle patterns corresponding to different seeds were registered. The samples were illuminated with an expanded and attenuated 10 $\mathrm{mW}$ He-Ne laser. The speckle images were then registered by a CCD camera, digitized to 8 bits by a frame grabber and stored in the memory of a personal computer.
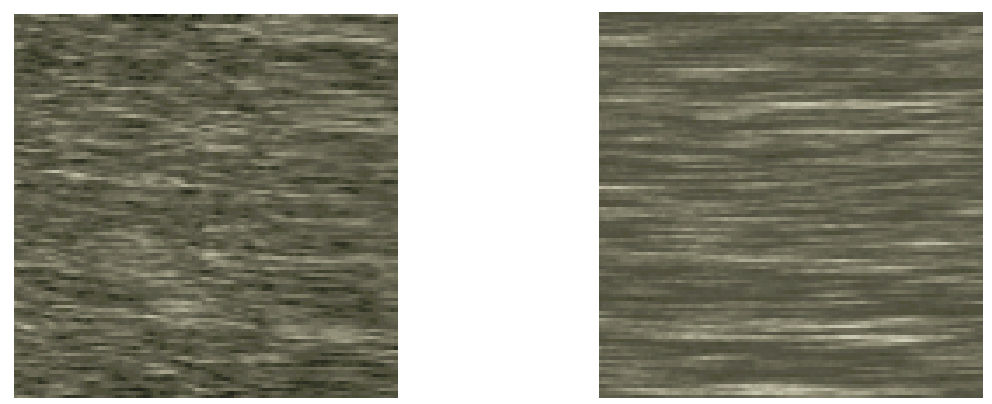

Fig. 1. (a) Dynamic speckles corresponding to a seed "alive" (b) Dynamic speckles corresponding to a seed "dead"

To recorded the time evolution of a speckle pattern we used the Oulamara et al. method [4]. It is, for every state of the phenomenon being assessed, 512 successive images were registered of the dynamical speckle pattern and a certain column was selected in each of them. With the selected column, a new $512 \times 512$ pixel $^{2}$ composite 
image was then constructed. In this image, named the time history of the speckle pattern (THSP), the rows represent different points on the object and the columns their intensity state in every sampled instant.

The activity of the sample appears as intensity changes in the horizontal direction. So, when a phenomenon shows low activity (died seeds), time variations of the speckle pattern are slow and the THSP shows elongated shape. When the phenomenon is very active (alive seeds), the THSP resembles an ordinary (spatial) speckle pattern as shown in Fig. 1.

\section{Difference Histogram Method}

The difference histogram of the image $Y[i, j]$ is the histogram of the auxiliary image, that is obtained by subtracting the original image of its replica, with a relative displacement of $[d 1, d 2]$ among them.

$$
Y_{d_{1}, d_{2}}^{(d)}[i, j]=Y\left[i+d_{1}, j+d_{2}\right]-Y[i, j]
$$

The classification method is completed by applying the decision Bayesian rule and by assuming a multinomial distribution law for the histograms values $x=\left\{x_{1}, x_{N}\right\}$ [7].

The image whose histogram is $x$, belongs to the class " $\mathrm{i}$ " if:

$$
U_{i}(x)=\min \left\{U_{j}(x)\right\}, j=1, \ldots . ., k
$$

where:

$$
U_{j}(x)=-\sum_{i=1}^{N} x_{i} \log \left[P_{j i}\right]
$$

where $P_{j i}$ is the probability that the HD takes the value $x_{i}$ for the class " $j$ ". These values are obtained from the HD patterns, which are representative of the $k$ classes.

In this experiment we have only one class: "dead", and for this reason it was necessary to use the parameter $U_{j}$ not to discriminate between some classes but to define a discrimination threshold between "dead" and "not dead" or "alive" textures. 


\section{Classification Procedure. Results}

Four groups of THSP images of seeds, corresponding to different levels of humidity were conformed. From each humidity group of 54 samples, a THSP image was picked up as the most representative of the "dead" state, according to its textural characteristics. This image texture was considered as the pattern corresponding to the "dead" class.

It was selected an subset of 10 THSP images textures with heterogeneous character (five of them corresponding to seeds qualified as "alive" and the other five as "dead" by a conventional method). The values of the Unser discrimination function related to the corresponding pattern were computed for this subset. These values were used to define a discrimination threshold between "alive" and "dead" textures for each humidity group. The heterogeneous character of the sample subset contributes to fit the threshold in a more precise way.

For classification process, the value of the discrimination function of each sample was compared to the threshold in order to classify the seeds in viable and not viable. The results were compared with those obtained by the conventional method.
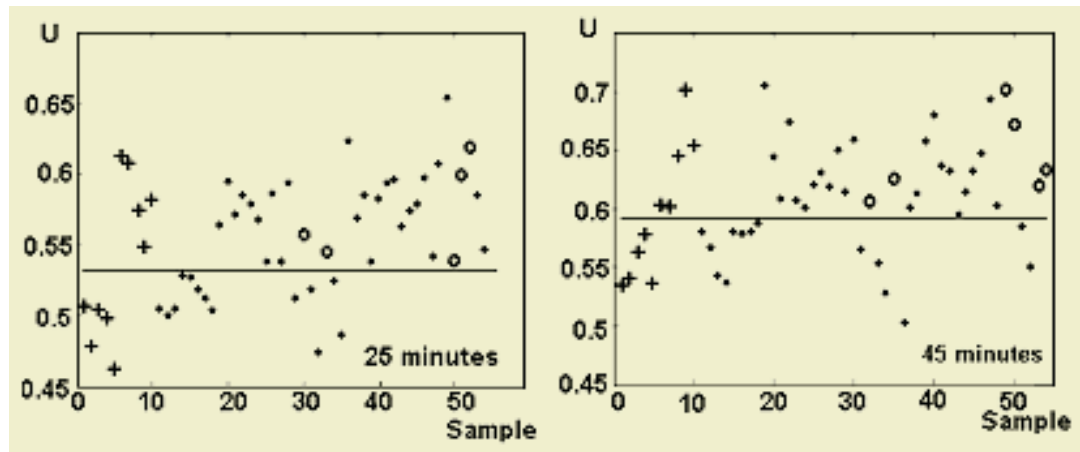

Fig. 2. The figure 2 shows the classification results for two levels of humidity. The value of the discrimination function $\mathrm{Uj}$ related to the pattern is plotted for each THSP image.

The straight line represents the threshold value of the discrimination function. For our method all results located under this line belong to the "dead" or non viable class. The crosses correspond to the sample subset that was considered to define the threshold value. The asterisks correspond to coincident classifications according to both methods: this method and the conventional one, and the circles correspond to non-coincident classifications.

The percent of coincident classifications was $85 \%, 91 \%, 92 \%$ and $90 \%$ for times of drying of 15, 25, 35 and 45 minutes respectively. 


\section{Conclusions}

According to the obtained results the proposed method revealed to be appropriate for the task of classification of seeds. In the case of the highest humidity level the disagreement between our method and the conventional one was the greatest. It should be said that in this case the analyzed images were noisier.

The characterization process of seeds done in this work, by using dynamic speckles and image processing, constitutes a less subjective method than the traditional ones and allows the automation of the process, increasing its efficiency.

The proposed method could be applied, not only to classification of seeds but also to any other phenomenon where the mean speckle lifetime be a significant measure of some type of sample activity, making possible the characterization of the temporary evolution of the phenomena.

\section{References}

1 Dainty, J.C. (ed.) : Laser speckle and related phenomena, Springer-Verlag, Berlin (1975).

3 Braga R. Et al.:,Proc. SPIE 4419, Tandil, Argentina (2001) 34-37.

2 Fernández, M.,Mavilio, A.,Rabal, H., Trivi,M.: Proc. SPIE 4419, Tandil, Argentina (2001) 178-181.

4 G. Oulamara et al.: Journal of Modern Optics, Vol.36(1989), 165-179.

5 Haralick R. M.: Proc. IEEE., Vol. 67, No. 5(1979) p. 786.

6 Haralick R. M.: IEEE Trans. Syst., Man, Cybern., Vol. SMC-3, No. 6(1973), p. 610.

7 Unser M.: IEEE Trans. Pattern Anal. Mach. Intell., Vol 8, No.1 (1986).p 118,

8 Tejera, M., Mavilio, A., Fernández, M.: Dimensiones Promediadas como descriptores de la textura. Mem. Foro Iberoam. Trat. Dig. Imag., Valencia, España, Oct. (1996 ).

9 Weldon T. P. y Higgins W. E. :Proc. ICIP-94, Austin, ,Texas, T-II(1994), p 620.

10 Palisson P.: Proc. ICIP-94, Austin, ,Texas, T-II, (1994) p 625.

11 Chang T., and Kuo,C.C.J.: Proc. Int. Conf. Acoust. Speech, Signal Proc, Vol. 3, (1992) pp. 661-664. 